\title{
Analysis of partial discharge Ultrasonic wave characteristic on typical Defects in GIS
}

\author{
Jing Gang YANG ${ }^{1, a}$, Wen SHI ${ }^{2}$, Hong Tao LI, Bin GONG ${ }^{1}$, Wei Yi JIANG ${ }^{1}$ \\ 1 Jiangsu Electric Power Corporation, Nanjing 211103, Jiangsu , China \\ ${ }^{2}$ Guodian nanjing automation co., LTD ,Nanjing 211106, Jiangsu, China
}

\begin{abstract}
For analyzing partial discharge ultrasonic wave characteristic on typical defects in SF6 gas insulation switchgear (GIS), a $110 \mathrm{kV}$ GIS was taken as an object to design and simulate the typical partial discharge models in GIS. There are point electrode model on bus and cavity of GIS, floating electrode model, air interstice model in insulator, metal particle and iron core electromagnetic shock models. PD signals on different defects were thus detected with the ultrasonic method. The experimental results indicated that the ultrasonic signals of different defects showed different characteristics in waveform, amplitude and phase resolved partial discharge chart, which is helpful in PD pattern recognition for GIS.
\end{abstract}

\section{Introduction}

Please submit sources files directly to the conference organizer. If the conference editors chose to provide print-ready PDF documents to the publisher, you have to submit high-resolution PDF file with all fonts embedded (see PDF guidelines) instead of the sources files. In this case, please remember that no final corrections will be made by the publisher.

Gas insulation switchgear (GIS) as an important equipment in the power system operation, in the event of failure, will cause huge economic losses and adverse social impact. Therefore, the operation reliability of the GIS is very important. The GIS internal insulation defect of the vast majority comes from the development of partial discharge, so the detection of partial discharge is an effective means of preventing GIS insulation fault ${ }^{[1-3]}$.

Ultrasound is one of the hot methods of domestic and foreign research of GIS partial discharge ${ }^{[4]}$, this method diagnosis of GIS by detecting partial discharge for ultrasonic signal, , so as to realize the accurate extraction of partial discharge signals, can be more comprehensive research intrinsic characteristics of partial discharge ${ }^{[5]}$.

\section{Utrasonic detection of partial discharge}

The final printed area will be $172 \times 252 \mathrm{~mm}$. In partial discharge in GIS can be induced in charge neutralization process, and the corresponding produce steep pulse current, the effect of electric current pulse will make the local area which occurs the partial discharge expanding and instantaneous heat, forming a similar explosion, the expansion of the heated region back to the original size after discharge, the density of the medium in an instant change caused by the partial discharge which produced a higher shrinkage volume change, form the ultrasound, in the form of spherical wave from the partial discharge point spread to all around, so when partial discharge occurs is also accompanied by ultrasound. On the other hand, ultrasound can also detect mechanical wave produced by the particle motion, on general engineering application of the ultrasonic to distinguish the motion of particles.

Combines both the ultrasonic method and digital measurement, this paper aims to detect partial discharge model typical discharge or free particles to beat the ultrasonic signal, after digital processing, spectra and statistics can be obtained, for further study of different defect types of partial discharge characteristics to provide data and test basis.

\section{Typical flaws with the design of the detection system}

GIS internal reason of the failure of tend to be various, in the manufacturing and assembly process, GIS will inevitably leave some minor defects. According to the relevant literature and the experience of field test, Produced high-voltage bus for a metal spike, wall bulging metal spikes, structure as shown in figure 1 .

\footnotetext{
a Corresponding author: 15105168828@163.com
} 


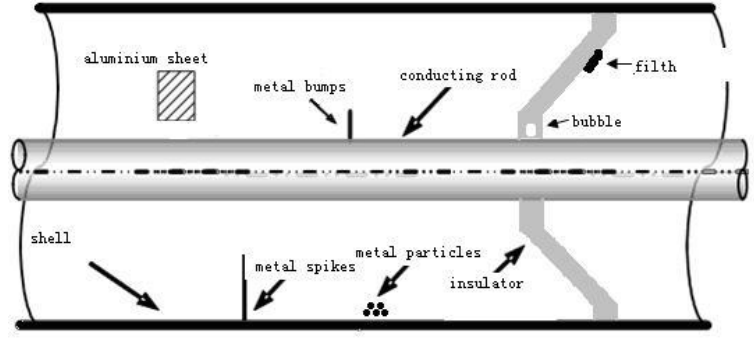

Figure 1. GIS common defects schematic

Five kinds of common defects of GIS model designed, including a high-voltage bus metal spike, GIS wall metal spike, floating potential, insulator within the air gap and free metal particles etc. The metal spike model is a steel needle which is $53 \mathrm{~mm}$ length, the equivalent radius of curvature of the cutting edge is $100 \mu \mathrm{m}$; Metal suspended potential defects by polyethylene insulation screw to fix the metal aluminium on the high voltage bus bar, the two are separated by a distance (test respectively use $1 \mathrm{~mm}, 1.5 \mathrm{~mm}, 2 \mathrm{~mm}$ and $4 \mathrm{~mm}$ gap distance), insulating screw can be used to adjust the clearance between guide rod and suspended aluminum distance.

Typical defects of GIS experiment and testing system of ultrasonic signal main structure schematic is shown in figure 2. The system is mainly composed of the maximum output voltage is $250 \mathrm{kV}$ AC testing transformer which without partial discharge, protection resistance $\mathrm{Rz}$ (water resistance), coupling capacitance $\mathrm{Ck}$ $(85 \mathrm{pF})$, detection impedance $(50 \Omega), \mathrm{C} 1$ and $\mathrm{C} 0$ divider, $110 \mathrm{kV}$ gis test section which built-in defect can be replaced $(\mathrm{Cx})$, AIA ultrasonic signal detector and pulse current method of detection system. Pulse current method as an auxiliary means, is used to compare and confirm collection for discharge signals.

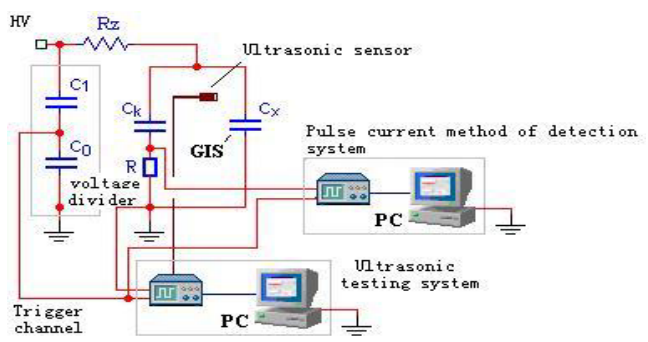

Figure 2. Test system structure diagram

Install the defects in GIS cavity in the body and the SF6 gas charging $0.45 \mathrm{MPa}$ when test.

\section{Partial discharge characteristics of defects}

\subsection{Busbar metal spike defects}

Due to reasons such as processing or installation, the GIS may form metal protrusions on high voltage busbar, spike part of its easy to form a strong field intensity area that can make the surrounding gas ionization, then producing partial discharge, its long-term effects can destroy GIS insulation condition ${ }^{[6]}$.
When test, the voltage from low to high, until stable the measured ultrasonic signal, record its initial discharge and strong discharge PRPD (phase - resolved partial discharge, partial discharge signals phase map) spectra, as shown in figure 3 .

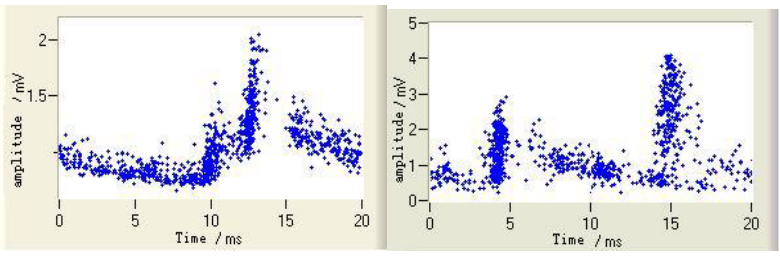

(a) starting discharge stage (b) strong discharge stage

Figure 3. Initial discharge and strong discharge PRPD spectra

From PRPD spectra can be found in this type of discharge in two stages: only negative half cycle of power frequency appear a small amount of discharge pulse and the positive half cycle basic not discharge during low voltage, the cause of this phenomenon is that the positive polarity, tip quality of positive ions ionized to larger then moving slowly, but electrons to cutting-edge mobile quickly. At high voltage, gas ionization is very intense when the positive half cycle power frequency, a lot of positive ions to be near plate equivalent reduced the plate spacing, Continue to ionization and form electron avalanche discharge and lingers, which may arise in the positive half cycle breakdown produces large amplitude of discharge pulses. Partial discharge level increased, the positive half cycle also appeared a lot of discharge pulse, and the positive and negative half cycle of discharge pulse in power frequency phase peak near the positive and negative.

\subsection{GIS wall metal spike defects}

GIS shell wall connected to the earth, but due to the presence of metal spikes and make the electric field distortion, the spikes in the electric field makes the SF6 gas ionization occurred around, causing partial discharge. When the experiment, the applied voltage from low to high, recording the initial discharge and strong discharge PRPD spectra as shown in figure 4.

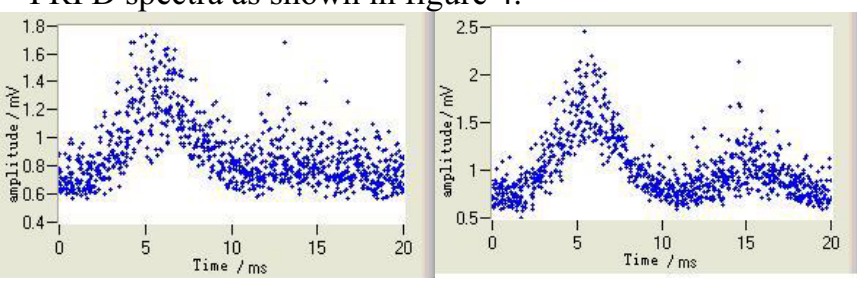

(a) starting discharge stage (b) strong discharge stage

Figure 4. Initial discharge and discharge strong PRPD spectra

It can be see that signal mainly concentrated in the positive half exemption value nearby when the initial discharge, as voltage continues to rise, discharge and discharge frequency continues to increase, and the negative half cycle also appeared some discharge pulse, phase concentration near the $270^{\circ}$.

When the metal spikes, therefore, the location is not at the same time, the cause of the partial discharge PRPD spectra will present different characteristics due to the 
polarity effect, in the low voltage, phase spectra show unimodal pattern, when the voltage is higher, two phase spectrum of discharge of the graph is bimodal pattern, but the amplitude and density of the signal distribution difference.

\subsection{GIS built-in suspension potential defects}

Found in the process of experiment, the size of the suspended electrode gap distance on the initial discharge voltage and discharge repetition rate has a significant impact, but little impact spectra characteristics of discharge, so this article only to the discharge gap distance to the defect of $2 \mathrm{~mm}$ model data for analysis.

Suspended potential defects caused by partial discharge is in the nature of the local breakdown. Because floating potential and high voltage bus form a capacitor, when both ends of the equivalent capacitance voltage exceeds the withstand voltage, high voltage busbar and gaps between floating electrode was breakdown, partial discharge. When the applied voltage is higher, defects in a power frequency cycle may occur many times charge discharge process, appear many times breakdown phenomenon. Records of initial discharge and strong discharge PRPD spectrum diagram as shown in figure 5 .
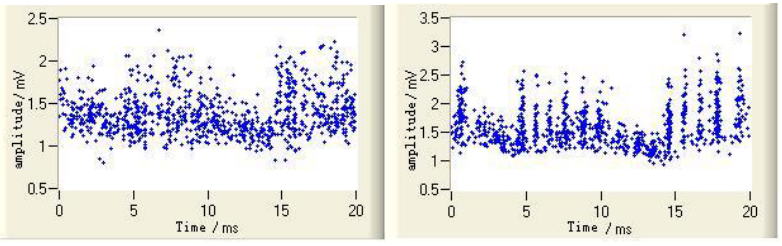

(a) starting discharge stage (b) strong discharge stage

Figure 5. Iinitial discharge and discharge strong PRPD spectra

\subsection{The basin insulator internal air bubble defect}

Solid dielectric in high voltage electrical equipment for insulation effect and mechanical strength of equipment, solid insulation gas gap (bubble) is the most common type of defect, when solid insulation appear dielectric defects high voltage equipment insulation will breakdown, causing serious accidents.

The experiment results show that the bubble size has a certain influence on the initial discharge voltage and discharge repeat rate, but has less effect on the discharge signal characteristics, so this article choose size $2 \mathrm{~mm}$ (thickness) x $1 \mathrm{~mm}$ (diameter) of the bubble experiment results to analyzing.

Considering the dispersivity of the discharge and the discharge mode of time accumulated, experiments on samples can step up to voltage until the first discharge signals collected, then the stability of this voltage applied of 10 minutes, and then gradually increase the voltage, each level voltages remain for 10 minutes or so on the discharge phenomenon. Record the starting discharge and strong discharge PRPD spectrum diagram as shown in figure 6 .
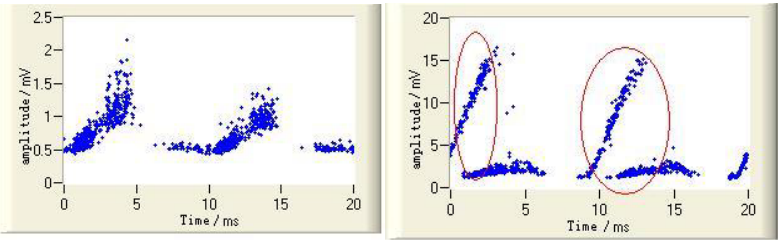

(a) starting discharge stage (b) strong discharge stage

Figure 6. Initial discharge and discharge strong PRPD spectra

PRPD spectra showed obvious phenomenon of "rabbit ear" (in part). Due to near zero air gap applied electric field in applied voltage polarity reversal, and the air gap within the dual pole field intensity in the same direction, the superposition of two field intensity in the air gap within the field strength increase, and discharge, so the "rabbit ear" signal spectra.

With the increase of applied voltage, "rabbit ear" part becomes more obvious, the spectra of the air gap discharge mode change is not big, generally considered as the partial discharge, air gap of defects are happening gas molecules within the discharge decomposition, produce the electronegativity molecules, such as $\mathrm{CO}_{2}$, $\mathrm{H}_{2} \mathrm{O}$, on the one hand these molecules influence the gases in the air gap discharge effect, on the other hand, with medium produces chemical reaction, generate crystalline objects attached in the surface of the medium, change the conductivity on the surface of the medium. Constant bombardment due to discharge of molecular and electronic media surface, the medium surface roughness increases, the influence on the discharge space charge accumulation, lead to the change of discharge spectra. Comparing the spectra of different voltages can also be found that with the increase of applied voltage and the phase of "rabbit ear" followed wide.

\subsection{Free metal particles defect}

Free particle impact cavity and partial discharge occurs in flight will produce ultrasonic, this article only on the particles of a study on ultrasonic features of partial discharge.

The experiment results show that the material, the size of the metal particles will affect the intensity of particles to beat, the smaller size, The lighter material, the more light beat the strong, but in the event of beating, the ultrasonic signal features are basically identical, so this article choose the experiment results of aluminum particles which diameter is $2 \mathrm{~mm}$ to analyze.

Recorded the initial discharge PRPD spectra and flight figure as shown in figure 7.

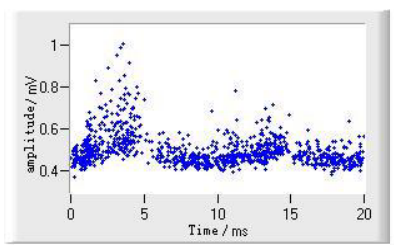

(a) PRPD spectra

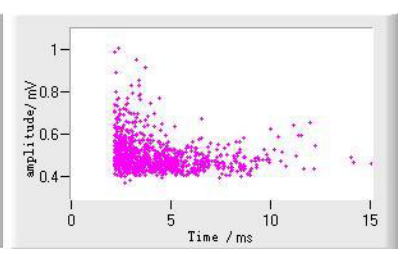

(b) flight figure
Figure 7. start discharging the PRPD spectra, flight figure 
Initial discharge, the signal is concentrated in the power supply voltage is near the peak, the signal amplitude is smaller, particle flight jitter is not active, discharge and discharge time interval between the statistics are not relevant, no obvious characteristics on the drawing in the flight. This is because when the voltage is not high, the metal particles are not violent beating, a model is equivalent to a wall in metal bumps defect model, at this point the first discharge signals appeared in the positive peak of the power supply.

With the applied voltage rise gradually, the particles gradually occur beating, discharge more and more intense, recorded the PRPD spectra and flight diagram of the starting discharge, as shown in figure 8.

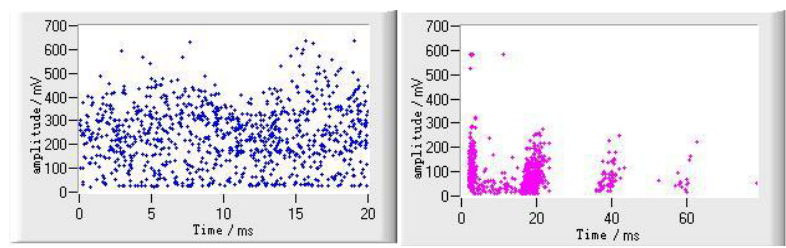

(a) PRPD spectra $\quad$ (b) flight figure

Figure 8. metal particle movement PRPD spectra, flight figure

Ultrasonic pulse signal when the free particle free beats more evenly distributed in $0 \sim 360^{\circ}$ phase cycle, more than $90 \%$ of the measurement results are similar, so it can be said that the free movement of particles in the free beats of large signal amplitude and no obvious phase correlation is one big characteristic. Although particle collision shell moment has nothing to do with the power frequency phase, but ultrasonic signal amplitude, time of flight are showing strong cyclical change, the flying time is about $20 \mathrm{~ms}$.

This is because when particles collide with the metal case, Immediately get to have a new charge up charge force upward movement, if ignore flight discharge lead to loss of charge occurs, an odd number of times after half cycle power frequency particles will receive a charge in the opposite direction, when the maximum charge force and gravity in the same direction (pointing to the electrode), the impact has great momentum to detect ultrasonic signal also reached maximum, so the flight figure carrying flight time and signal amplitude comparison of regular periodic variation characteristics.

\section{Discussion}

Through this study, can get the following typical defects of GIS partial discharge of ultrasonic signal features:

1) metal spike defects : When the metal spikes in different places, its cause partial discharge PRPD spectra will present different characteristics due to the polarity effect, in the low voltage, phase spectra show unimodal pattern, the high voltage, phase spectrum of two kinds of discharge the graph is bimodal pattern, but the signal amplitude and the signal density distribution difference;

2) suspension potential defects: partial discharge is mainly focused on the falling edge of the power supply voltage, as the voltage continues to rise, phase appears to have the phenomenon of zero drift, and appear the phenomenon of multiple discharge - discharge extinction - discharge;

3) Insulator internal air bubble defect: the biggest feature of the air bubble defect is in positive and negative half cycle rise along their "rabbit ear" shape distribution, and with the increase of voltage "rabbit ear" amplitude increases, the trend of "rabbit ear" starting phase decreases;

4) Free metal particles defect: particles without movement, the equivalent of the spikes defect on the grounding body, partial discharge signals show its discharge characteristics of thorns; Discharge occurs When the particle movement, defect discharge occurs in the whole power frequency cycle, power frequency ultrasonic signal amplitude, time of flight but showed strong cyclical changes;

GIS partial discharge is a complicated process, encountered in the process of ultrasonic wave in the spread of disorder will produce a series of reflection and refraction, so using ultrasonic wave method to detect internal defects of GIS still needs further research and exploration.

\section{References}

1. Schei A, Kyrkjeeide S, Larsen V. Asia Pacific. IEEE, Yokohama, 919-924 (2002).

2. Li Da-jian,Liang Ji-zhong,Bu Ke-wei,Yang Jinggang,Li Yan-ming.High Voltage Apparatus,45(1):7275 (2009).

3. Jin Yi, Liu Wei, Zha Xian-guang, et.Jiangsu Electrical Engineering, 31(2):12-15 (2012).

4. Lundgaard LE. Partial discharge XIII. IEEE Electrical Insulation Magazine, 8 (4): 10.1109/1157.145095 (1992).

5. Wang Jianming, LI Pen.Jiangsu Electrical Engineering, 31(6): 62-65 (2013).

6. Zhou Yun-feng, Zhang Zi-yang, Yang Jinggang,et.Jiangsu Electrical Engineering,31(4):3-5 (2012). 